\title{
AURC Infinity Predicted Normalized by
}

\section{Surface Area}

National Cancer Institute

\section{Source}

National Cancer Institute. AURC Infinity Predicted Normalized by Surface Area. NCI

Thesaurus. Code C92360.

The area under the urinary excretion rate curve (AURC) extrapolated to infinity, based on the last predicted excretion rate, divided by the surface area. 